RESEARCH ARTICLE

\title{
Elevated Hemoglobin A1C is Significantly Associated with Decreased Post-Exercise Heart Rate Recovery in Patients with Diabetes Mellitus
}

\author{
Hasan Hareem $^{1 *}$, Everman Sarah ${ }^{2}$, Alawi Alsheikh Ali ${ }^{3}$, Farooqi M Hamed ${ }^{1}$, Blanchon Bruno ${ }^{1}$ and \\ Hassoun Ahmed ${ }^{1}$
}

${ }^{1}$ Dubai Diabetes Center, Dubai, United Arab Emirates

${ }^{2}$ College of Graduate Health Studies, A T Still University, USA

${ }^{3}$ College of Medicine, Mohammed Bin Rashid University of Medicine and Health Sciences,

Dubai, United Arab Emirates

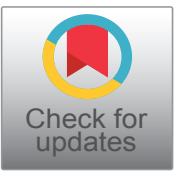

*Corresponding author: Hareem Hasan, Dubai Diabetes Center, Al-Hudaiba Awards Building, First Floor, Block-C, Second December Street, Diyafa, Dubai, United Arab Emirates, Postal Code: 215252, Tel: 009-714-381-9665, Fax: 009-714-3546669,E-mail: hhasan@dha.gov.ae; hareemsaadat@yahoo.com

\begin{abstract}
Heart Rate Recovery (HRR) has been associated with cardiovascular and all-cause mortality in both healthy individuals and those with diabetes. We examined the association between Hemoglobin A1C ( $\mathrm{HbA} 1 \mathrm{c})$ and post-exercise HRR in patients with diabetes. Participants who were free from known cardiac disease or severe orthopedic problems referred to an exercise specialist for a fitness assessment. All participants underwent a graded exercise test using a standard Balke-ware protocol. HRR defined as the decrease in heart rate from peak exercise after one minute of a cool down walk at $2 \mathrm{mph}$ and $0 \%$ incline. The most recent $\mathrm{HbA1c}$ (within three months) recorded for each patient. This study included 411 individuals with diabetes [age $45 \pm 10$, $269(65 \%)$ males, $375(91 \%)$ with type 2 diabetes, BMI 31 $\pm 6,49 \%$ with $\mathrm{BMI}>30 \mathrm{~kg} / \mathrm{m}^{2}$ ]. The mean $\mathrm{HbA} 1 \mathrm{c}$ was $7.3 \%$ $\pm 1.6(56 \mathrm{mmol} / \mathrm{mol})$. The participants divided into 4 equal cohorts based on the quartiles of HRR. Using multiple logistic regression, adjusting for age, gender, BMI, metabolic equivalent, beta blocker usage, hypertension, and duration of diabetes, there was a significant association between $\mathrm{HbA} 1 \mathrm{c}$ and odds of having a HRR in the lowest quartile, such that for every unit increase in $\mathrm{HbA} 1 \mathrm{c}$, there was $13 \%$ increase in odds of being in the lowest HRR quartile (adjusted odds ratio 1.125 [1.002, 1.264]). Among diabetic patients without known cardiac disease, poor glycemic control as measured by elevated $\mathrm{HbA} 1 \mathrm{c}$ is significantly associated with decreased post-exercise HRR.
\end{abstract}

\section{Keywords}

Diabetes, Exercise capacity, Glucose control, Heart rate recovery, Hemoglobin A1C

\section{Introduction}

The prevalence and incidence of diabetes mellitus has been increasing all over the world. International Diabetes Federation (IDF) estimated in 2015 that there were 415 million adults aged 20-79 living with diabetes worldwide, including 193 million who were undiagnosed. These trends are not projected to decrease over the next several years. The prevalence of diabetes is very high throughout the Middle East region and estimated around $14.6 \%$ in the United Arab Emirates [1]. Almost half of the adult patients attending primary health centers had undiagnosed diabetes or increased diabetes risk [2].

Autonomic neuropathy is a common complication of diabetes mellitus. Autonomic dysfunction has been associated with all-cause mortality, cardiac disease morbidity, and mortality in diabetes $[3,4]$. Heart Rate Variability (HRV) and Heart Rate Recovery (HRR) both are the measures of autonomic nervous system function. Heart Rate Recovery (HRR) immediately after exercise is a measure of sympathetic and parasympathetic function of the heart [5] whereas, HRV is a physiological variation in the interval between heartbeats and is regulated by the interaction of the sympathetic and parasympathetic tone [6]. 
The association of HRV and Hemoglobin A1C (HbA1c) has been studied in individuals with diabetes in several studies. A dose-response relationship was reported between $\mathrm{HbA} 1 \mathrm{c}$ and the measures of overall reduced HRV. The studies concluded that chronic and worsening hyperglycemia causes a decrease in all parameters of HRV that determine early cardiac autonomic dysfunction and parasympathetic loss [7-10]. It has been previously reported that abnormal HRR is associated with impaired glucose tolerance and increasing fasting blood glucose [11-13]. However, the association between abnormal HRR and increased level of Hemoglobin A1C (HbA1c) among people with diabetes is not well-studied and needing further research. Therefore, the purpose of this study was to determine the association between elevated $\mathrm{HbA} 1 \mathrm{c}$ level and the post-exercise HRR as a measure of post-exercise parasympathetic reactivation.

\section{Materials and Methods}

\section{Subjects}

This study included 479 patients with diabetes referred to an exercise specialist for a graded exercise test to assess their exercise capacity at Dubai Diabetes Center. Of the 479 patients, 68 (14\%) were excluded because of the following reasons: They had a history of coronary artery disease, pacemakers, arrhythmias, atrial fibrillation, cardiac failure, myocardial infarction, severe orthopedic problems, or failure to achieve at least 85 percent of their age-determined maximum heart rate during the exercise test. Written consent was obtained from all participants and the institutional ethics committee approved this study with registration number DSREC-02/2016_04.

A detailed medical history, family and drug history, exercise habits, and smoking habits were obtained from all participants. Systolic and diastolic blood pressure, height, weight, Body Mass Index (BMI), blood glucose, $\mathrm{HbA1c}$, and lipid profile were measured. Participants underwent a graded exercise tolerance test on a treadmill and exercise capacity was calculated as total Metabolic Equivalents (METs) achieved at peak exercise (one MET $=3.5 \mathrm{ml}$ of oxygen uptake per $\mathrm{kg}$ of body weight per minute). Diabetes was confirmed if the measured fasting blood glucose was equal to or greater than 126 $\mathrm{mg} / \mathrm{dl}$ at least twice or if any anti-hyperglycemic agents have been used [14]. Body mass index was calculated as the ratio of weight $(\mathrm{kg})$ to standing height $(\mathrm{m})$ squared $\left(\mathrm{kg} / \mathrm{m}^{2}\right)$. Normal, over weight and obese were categorized according to the World Health Organization (WHO) criteria as BMI 18.5-24.99, $\geq 25$, and $\geq 30 \mathrm{~kg} / \mathrm{m}^{2}$, respectively.

\section{Treadmill exercise test}

All participants performed a graded treadmill exercise test on an electronic treadmill (GE CASE Exercise Testing System) according to the Balke-ware protocol in which speed remains constant at $3.3 \mathrm{mph}$ and inclina- tion was increased by $1 \%$ each minute for 25 minutes. Electrocardiogram and heart rate were monitored continuously. Patients were encouraged to exercise until they reach at least $85 \%$ of their age-predicted maximum heart rate. The Borg 6-20 RPE scale was used to assess the perceived exertion [15]. On the Borg 6-20 RPE scale, an estimation of RPE 15 (verbal anchor = hard) was considered as the optimal limit, an esteem proposed to demonstrate a higher probability of exceeding anaerobic threshold $[16,17]$. Blood pressure and RPE were assessed at the end of each stage.

The following exercise test termination criteria was used: Failure to reach at least $85 \%$ of age-predicted maximum heart rate when patient request to stop or inability to keep up with the treadmill, systolic blood pressure $>250 \mathrm{~mm}$ of $\mathrm{Hg}$, typical chest discomfort, severe arrhythmias, more than $1 \mathrm{~mm}$ of horizontal or down-sloping ST segment depression, and/or technical difficulties. If a patient had ST depression more than 1 $\mathrm{mm}$ or exercise-induced angina/arrhythmias, they were not included in the data analysis. During each stage and recovery period, symptoms such as chest discomfort, shortness of breath, fatigue, dizziness, leg pain, heart palpitations, blood pressure, heart rate, cardiac rhythm, and exercise workload in METs was recorded. Predicted peak heart rate was calculated as 220 minus the patient's age.

\section{HbA1c test}

Hemoglobin A1C was measured using the DCA 2000 + system (Bayer, Germany). The most recent HbA1c value (within three-month time) was recorded for each patient.

\section{Heart rate recovery}

HRR is defined as reduction in the heart rate at peak exercise to that measured after one minute of recovery. After achieving at least 85 percent of age-determined heart rate, all patients spent one minute walking in the cool-down or recovery period at the speed of $2 \mathrm{mph}$ and $0 \%$ incline and a cut-off value of less than or equal to 12 bpm as abnormal based on the previous studies.

\section{Medications}

In this study, we did not require the participants to stop any medications including beta-blockers. A very small percentage of our group was on beta-blockers (5\%).

\section{Statistical analysis}

The study population was divided into four near-equal cohorts based on the quartiles of HRR. Continuous variables were summarized as mean \pm standard deviation and compared using ANOVA. Categorical variables were summarized as percentages and compared using the Chi square test. Logistic regression was used to estimate the association between $\mathrm{HbAlc}$ and 
Table 1: Baseline characteristics by quartiles of heart rate recovery.

\begin{tabular}{|c|c|c|c|c|c|c|}
\hline Variable & $\begin{array}{l}\text { All Subjects } \\
(\mathrm{N}=411)\end{array}$ & $\begin{array}{l}\text { HRR First } \\
\text { Quartile } \\
(\mathrm{n}=96)\end{array}$ & $\begin{array}{l}\text { HRR Second } \\
\text { Quartile } \\
(n=93)\end{array}$ & $\begin{array}{l}\text { HRR Third } \\
\text { Quartile } \\
(n=108)\end{array}$ & $\begin{array}{l}\text { HRR Fourth } \\
\text { Quartile } \\
\text { (n=114) }\end{array}$ & $\mathbf{P}$ \\
\hline Age & $45 \pm 10$ & $47 \pm 10$ & $45 \pm 10$ & $46 \pm 10$ & $43 \pm 11$ & 0.0923 \\
\hline Male Gender (\%) & 65 & 62 & 71 & 69 & 61 & 0.2710 \\
\hline Heart Rate Recovery (bpm) & $22 \pm 8$ & $13 \pm 3$ & $18 \pm 1$ & $23 \pm 1$ & $31 \pm 5$ & $<0.0001$ \\
\hline Diabetes Duration (years) & $8 \pm 7$ & $9 \pm 7$ & $9 \pm 7$ & $8 \pm 6$ & $8 \pm 7$ & 0.4299 \\
\hline Diabetes Type (Type 2\%) & 91 & 90 & 92 & 93 & 86 & 0.2996 \\
\hline $\mathrm{HbA} 1 \mathrm{c} \%(\mathrm{mmol} / \mathrm{mol})$ & $\begin{array}{l}7.3 \pm 1.6 \\
(56)\end{array}$ & $\begin{array}{l}7.7 \pm 1.8 \\
(61)\end{array}$ & $\begin{array}{l}7.4 \pm 1.5 \\
(57)\end{array}$ & $\begin{array}{l}7.2 \pm 1.5 \\
(55)\end{array}$ & $\begin{array}{l}7.2 \pm 1.8 \\
(55)\end{array}$ & 0.0082 \\
\hline METS & $7.9 \pm 2$ & $6.5 \pm 2$ & $7.7 \pm 2$ & $8.6 \pm 2$ & $8.6 \pm 2$ & $<0.0001$ \\
\hline BMI & $31 \pm 6$ & $33 \pm 6$ & $32 \pm 5$ & $30 \pm 5$ & $29 \pm 5$ & $<0.0001$ \\
\hline Hypertension (\%) & 44 & 64 & 45 & 39 & 32 & $<0.0001$ \\
\hline Beta Blocker Use (\%) & 5 & 13 & 2 & 3 & 4 & 0.0026 \\
\hline Dyslipidemia (\%) & 84 & 83 & 86 & 87 & 79 & 0.2921 \\
\hline
\end{tabular}

Heart Rate Recovery first quartile $<17 \mathrm{bpm}$, second quartile $\geq 17$ and $<21 \mathrm{bpm}$, third quartile $\geq 21$ and $<26$ and fourth quartile $\geq 26$.

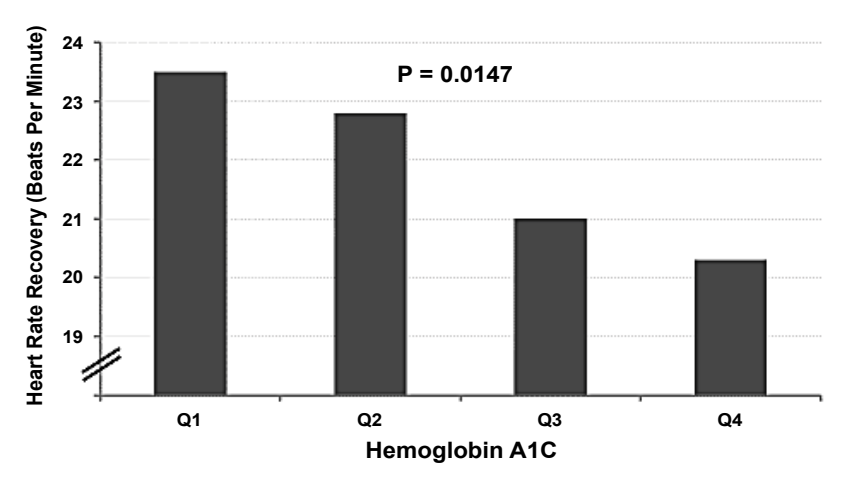

Figure 1: Relationship between hemoglobin $\mathrm{A} 1 \mathrm{C}$ and heart rate recovery $(\mathrm{HRR}=\mathrm{bmp})$ one minute after exercise. Quartiles of heart rate recovery: $\mathrm{Q} 1=\mathrm{HbA} 1 \mathrm{c}<6.2 ; \mathrm{Q} 2=$ $6.2 \leq \mathrm{HbA} 1 \mathrm{c}<7.0 ; \mathrm{Q} 3=7.0 \leq \mathrm{HbA} 1 \mathrm{c}<7.9 ; \mathrm{Q} 4=\mathrm{HbA} 1 \mathrm{c} \geq$ 7.9. $P_{\text {trend }}=0.0147$ across the quartiles.

HRR adjusting for age, gender, BMI, metabolic equivalent, beta-blocker usage, hypertension, and duration of diabetes. Odds ratio with $95 \%$ confidence interval was calculated for the adjusted association. An interaction term was introduced in the logistic model to assess if the association between $\mathrm{HbA1C}$ and HRR was modified by BMI. A P-value of $<0.05$ was considered statistically significant.

\section{Results}

The mean $\mathrm{HbA} 1 \mathrm{c}$ was $7.3 \pm 1.6$ and, $49 \%$ of the patients had a value $>7$ percent $(56 \mathrm{mmol} / \mathrm{mol})$. Adjusting for age, gender, BMI, beta blocker usage, metabolic equivalent, hypertension, and duration of diabetes, there was a significant association between $\mathrm{HbA1c}$ and odds of having a HRR in the lowest quartile, such that for every unit increase in $\mathrm{HbA1c}$, there was $13 \%$ increase in the odds of being in the lowest HRR quartile (adjusted odds ratio $1.125[1.002,1.264])$. Notably, the association between $\mathrm{HbA} 1 \mathrm{c}$ and $\mathrm{HRR}$ was modified by BMI ( $\mathrm{p}$ for interaction $=0.0043$ ), with a trend across the quartiles $\left(P_{\text {trend }}=0.0147\right)$. In univariate analysis, as HbA1c increased, HRR decreased (Figure 1). The adjusted association between $\mathrm{HbA1c}$ and HRR was significant among patients with $\mathrm{BMI}<30 \mathrm{~kg} / \mathrm{m}^{2}$ (adjusted odds ratio 1.197 $[1.010,1.418])$, but not among patients with BMI of $30 \mathrm{~kg} / \mathrm{m}^{2}$ or higher (adjusted odds ratio 1.049 [0.894, 1.231]).

Baseline characteristics and summary results are shown in Table 1. Compared to the patients in the lowest quartile of HRR, those in the highest quartile tended to be younger, with lower $\mathrm{HbA1c}$ and $\mathrm{BMI}$, have lower prevalence of hypertension and beta-blocker use, and a higher exercise capacity.

\section{Discussion}

This study showed that HbA1c is strongly associated with delayed HRR and that the adjusted association between $\mathrm{HbA1C}$ and HRR was particularly significant among patients with $\mathrm{BMI}<30 \mathrm{~kg} / \mathrm{m}^{2}$ (non-obese) compared to patients with BMI of $30 \mathrm{~kg} / \mathrm{m}^{2}$ or higher (obese). This somewhat deviates from a previous study that showed a negative correlation between slow HRR and HbA1c in obese patients with Type 2 diabetes [18]. However, in our study, we did not measure abdominal obesity or visceral fat, which might have influenced HRR among those with normal BMI if they had abdominal obesity despite a normal BMI [19]. Studies showed that the central fat in particular, abdominal visceral obesity was associated with impaired sympathetic nervous system function more than peripheral fat [20].

HRR is a measure of autonomic nervous system function $[4,5]$. During exercise, heart rate increases because of the sympathetic activation and vagal withdrawal. During recovery from exercise, vagal reactivation is responsible for quickly reducing the heart rate $[4,21]$. HRR is an independent predictor of death among healthy adults as well as among patients with diabetes. A delay in the reduction of heart rate after exercise is an indicator of impaired autonomic nervous system functioning, specifically reduced parasympathetic activity $[22,23]$. Decreased HRR is the preliminary sign of Cardiac Autonomic Neuropathy and subclinical diabetic au- 
tonomic neuropathy can appear one to two years after abnormality in the HRR [24]. Thus, HRR derived from a standard exercise may help to predict and diagnose the early stage of cardiovascular disease and Cardiac Autonomic Neuropathy (CAN) in patients with diabetes [25].

Abnormal HRR has been associated with insulin resistance, BMI, abdominal obesity, low HDL cholesterol and high triglycerides/HDL ratio $[26,27]$, metabolic syndrome [28], and high fasting plasma glucose [12,13,29] in healthy adults as well as in patients with diabetes $[3,4]$. Abnormal HRR has also been associated with endothelial dysfunction, myocardial ischemia, carotid atherosclerosis, cardiovascular events, and stroke in patients with diabetes [4,23,28,30].

HRR is a modifiable risk factor. Studies showed that HRR improves with weight loss, exercise training, and cardiac rehabilitation [18,21,31,32]. Burger, et al. [33] and the Diabetes Control and Complications (DCC) trial [34] found that stringent glycemic control may improve autonomic dysfunction and even a modest reduction in the mean $\mathrm{HbA} 1 \mathrm{c}$ produced favorable effects on Cardiac Autonomic Neuropathy (CAN). These authors demonstrated that the glycemic control was the only therapeutic approach that decreased the risk of progression of autonomic neuropathy and could possibly improve CAN. Autonomic neuropathy is reversible with good glycemic control in its early stages, but, in more advanced stages, there was a continued deterioration despite a decrease in blood glucose level [33].

Our current study adds to the aforementioned findings in several important ways. Elevated $\mathrm{HbA} 1 \mathrm{c}$ is strongly associated with abnormal HRR and there is a dose response relationship between worsening $\mathrm{HbA} 1 \mathrm{c}$ and delayed HRR. The results of our current study suggest that heart rate after one minute of recovery from treadmill exercise testing has clinical importance because of its association with autonomic imbalance. This could be useful to identify the patients with diabetes having high risk of developing autonomic neuropathy in future. This screening might identify those patients who are candidates for therapy directed at improving HRR or who need additional clinical evaluation. Further studies are needed to determine whether improvement in glycemic control will benefit the long-term autonomic function.

\section{Limitations}

There are some limitations of this study included that blood glucose measured on the test day was not the fasting blood glucose for all patients. Therefore, we were not be able to determine an association between fasting blood glucose and HRR, though this has been described in previous studies. In addition, this study did not measure insulin resistance, gas exchange, and abdominal adiposity.

\section{Conclusion}

This study showed that elevated $\mathrm{HbA} 1 \mathrm{c}$ is strongly associated with delayed HRR, which is a powerful indicator of all-cause and cardiovascular mortality in healthy people as well as in people with diabetes. We found that among patients with diabetes without known cardiac disease, poor glycemic control as measured by elevated $\mathrm{HbA1C}$ was significantly associated with decreased post-exercise HRR, especially among non-obese individuals. Previous studies showed a strong association between HRV and $\mathrm{HbA} 1 \mathrm{c}$ in patients with diabetes. Our study showed a similar dose response relationship between HRR and HbA1c. This study added an important finding to identify the patients with high risk of autonomic dysfunction and might help the patients in long-term autonomic function by improving their glycemic control and HRR.

\section{Conflict of Interest}

This manuscript has not been published previously in whole or in part. The authors have no conflicts of interest regarding this study. There is no financial support for this study.

\section{Acknowledgments}

A. Alsheikh Ali performed the statistical analysis, participated in the analysis interpretation, and critically revised the study protocol and article draft.

A. Hassoun and S. Everman appraised and revised the study protocol and article draft.

M. H. Farooqi and B. Blanchon commented on the study protocol and article draft.

$H$. Hasan conceived the study, wrote the protocol draft, performed the literature search, extracted the data and wrote the article draft.

All authors approved the final version of the article.

\section{Grant}

This research received no specific grant from any funding agency in the public, commercial or not-forprofit sectors.

\section{References}

1. (2015) International Diabetes Federation Diabetes Atlas. ( $7^{\text {th }}$ edn), Brussels, Belgium.

2. Saadi H, Al Kaabi J, Benbarka M, Khalili A, Almahmeed W, et al. (2010) Prevalence of undiagnosed diabetes and quality of care in diabetic patients followed at primary and tertiary clinics in Abu Dhabi, United Arab Emirates. Rev Diabet Stud 7: 293-302.

3. Cheng YJ, Lauer MS, Earnest CP, Church TS, Kampert JB, et al. (2003) Heart rate recovery following maximal exercise testing as a predictor of cardiovascular disease and all-cause mortality in men with diabetes. Diabetes Care 26: 2052-2057.

4. Chacko MK, Bauer AT, Dale AR, Dixon AJ, Schrier WR, et al. (2008) Heart rate recovery predicts mortality and cardiovascular events in patients with type 2 diabetes. Med Sci Sports Exerc 40: 288-295. 
5. Cole CR, Blackstone EH, Pashkow FJ, Snader CE, Lauer MS (1999) Heart rate recovery immediately after exercise as a predictor of mortality. $\mathrm{N}$ Engl $\mathrm{J}$ Med 341: 1351-1357.

6. Task Force of the European Society of Cardiology and the North American Society of Pacing and Electrophysiology (1996) Heart rate variability: Standards of measurement, physiological interpretation and clinical use. Circulation 93: 1043-1065.

7. Faulkner SM, Quinn L, Rimmer HJ, Rich HB (2005) Cardiovascular endurance and heart rate variability in adolescents with type 1 or type 2 diabetes. Biol Res Nurs 7: 16-29.

8. Jaiswal M, Fingerlin ET, Urbina ME, Wadwa PR, Talton WJ, et al. (2013) Impact of glycemic control on heart rate variability in youth with type 1 diabetes: The search CVD study. Diabetes Technol Ther 15: 977-983.

9. Mirza M, Lakshmi ANR (2012) A comparative study of heart rate variability in diabetic subjects and normal subjects. International Journal of Biomedical and Advanced Research 3: 640-644.

10. Singh PJ, Larson GM, O'Connell JC, Wilson PF, Tsuji H, et al. (2000) Association of hyperglycemia with reduced heart rate variability (The Framingham Heart Study). Am J Cardiol 86: 309-312.

11. Celebi OO, Celebi S, Canbay A, Gokaslan S, Diker E (2014) Impaired heart rate recovery in patients with impaired glucose tolerance. Acta Endo (BUC) 10: 76-83.

12. Panzer C, Lauer MS, Brieke A, Blackstone E, Hoofwerg $B$ (2002) Association of fasting plasma glucose with heart rate recovery in healthy adults: A population based study. Diabetes 51: 803-807.

13. Seshadri N, Acharya N, Lauer MS (2003) Association of diabetes mellitus with abnormal heart rate in patients without known coronary artery disease. The American Journal of Cardiology 91: 108-111.

14. Genuth S, Alberti KG, Bennett P (2003) Expert committee on the diagnosis and classification of diabetes mellitus, the expert committee on the diagnosis and classification of diabetes mellitus follow-up report on the diagnosis of diabetes mellitus. Diabetes Care 26: 3160-3167.

15. Borg GA (1982) Psychophysical bases of perceived exertion. Med Sci Sports Exerc 14: 377-381.

16. Fletcher FG, Balady JG, Amsterdam AE, Chaitman B, Ecke $R$, et al. (2001) Exercise standards for testing and training; A statement for healthcare professionals from the American Heart Association. Circulation 104: 1694-1740.

17. Pinkstaff S, Peberdy AM, Kontos CM, Finucane S, Arena R (2010) Quantifying exertion level during exercise stress testing using percentage of age-predicted maximal heart rate, rate pressure product and perceived exertion. Mayo Clin Proc 85: 1095-1100.

18. Fang YZ, Prins BJ, Sharman J, Marwick TH (2005) Determinants of exercise capacity in patients with type 2 diabetes. Diabetes Care 28: 1643-1648.

19. Fu X, Zhu F, Zhao X, Ma X, Zhu S (2014) Central fat accumulation associated with metabolic risks beyond total fat in normal BMI Chinese adults. Ann Nutr Metab 64: 93-100.
20. Beske DS, Alvarez EG, Ballard PT, Davy PK (2002) Reduced cardio vagal baroreflex gain in visceral obesity: Implications for the metabolic syndrome. Am J Physiol Heart Circ Physiol 282: 630-635.

21. Carnethon RM, Craft LL (2008) Autonomic regulation of the association between exercise and diabetes. Exerc Sport Sci Rev 36: 12-18.

22. Arai $Y$, Saul JP, Albrecht $P$, Hartley LH, Lilly LS, et al. (1989) Modulation of cardiac autonomic activity during and immediately after exercise. Am J Physiol 256: 132-141.

23. Georgoulias P, Demakopoulos N, Valotassiou V, Orfanakis A, Zaganides A, et al. (2009) Long-term prognostic value of heart rate recovery after treadmill testing in patients with diabetes mellitus. Int J Cardiol 134: 67-74.

24. Pourmoghaddas A, Moghaddasian A, Garakyaraghi M, Nezarat N, Mehrabi A (2013) Heart rate recovery in exercise test in diabetic patients with and without microalbuminuria. ARYA Atheroscler 9: 167-171.

25. Sacre WJ, Jellis LC, Coombes SJ, Marwick HT (2012) Diagnostic accuracy of heart-rate recovery after exercise in the assessment of diabetic cardiac autonomic neuropathy. Diabet Med 29: e312-e320.

26. Spies C, Otte C, Kanaya A, Pipkin SS, Schiller BN, et al. (2005) Association of metabolic syndrome with exercise capacity and heart rate recovery in patients with coronary heart disease in the heart and soul study. Am J Cardiol 95: 1175-1179.

27. Shishebor MH, Hougwerf BJ, Lauer MS (2004) Association of triglycerides to HDL cholesterol ratio with heart rate recovery. Diabetes Care 27: 936-941.

28. Kim J, Byun W, Sui X, Lee D, Cheng JY, et al. (2011) Heart rate recovery after treadmill exercise testing is an independent predictor of stroke incidence in men with metabolic syndrome. Obes Res Clin Pract 5: e295-e303.

29. Sung J, Choi YH, Park JB (2006) Metabolic syndrome is associated with delayed heart rate recovery after exercise. J Korean Med Sci 21: 621-626.

30. Yamada T, Lee T, Yoshitama T, Saeki F, Makino K (2011) Heart rate recovery after exercise is a predictor of silent myocardial ischemia in patients with type 2 diabetes. Diabetes Care 34: 724-726.

31. Ribisl MP, Gaussoin AS, Lang W, Bahnson J, Connelly AS, et al. (2012) Lifestyle intervention improves heart rate recovery from exercise in adults with type 2 diabetes: Results from the Look Ahead Study. Journal of Obesity 2012: 1-12.

32. Tiukinhoy S, Beohar N, Hsie M (2003) Improvement in heart rate recovery after cardiac rehabilitation. J Cardiopulm Rehabil 23: 84-87.

33. Burger AJ, Weinrauch LA, D'Elia JA, Aronson D (1999) Effects of glycemic control on heart rate variability in type one diabetes patients with cardiac autonomic neuropathy. Am J Cardiol 84: 687-691.

34. The Diabetes Control and Complications Research Trial Group (1998) The effect of intensive diabetes therapy on measure of autonomic nervous system function in the Diabetes Control and Complications Trial (DCCT). Diabetologia 41: 416-423. 